\title{
Diacronie
}

Studi di Storia Contemporanea

$\mathrm{N}^{\circ} 28,4 \mid 2016$

La voce del silenzio

\section{Guido Panvini, Cattolici e violenza politica. L'altro album di famiglia del terrorismo italiano}

Jacopo Bassi e Luca Bufarale

\section{(2) OpenEdition}

Edizione digitale

URL: http://journals.openedition.org/diacronie/4662

DOI: $10.4000 /$ diacronie.4662

ISSN: 2038-0925

Editore

Association culturelle Diacronie

Notizia bibliografica digitale

Jacopo Bassi e Luca Bufarale, « Guido Panvini, Cattolici e violenza politica. L'altro album di famiglia del terrorismo italiano », Diacronie [Online], № 28, 4 | 2016, documento 30, Messo online il 29 décembre 2016, consultato il 24 septembre 2020. URL : http://journals.openedition.org/diacronie/4662 ; DOI : https://doi.org/10.4000/diacronie.4662 


\section{Diacronie}

N. 28 | 4|2016 La voce del silenzio: intelligence, spionaggio e conflitto nel XX secolo

30/

\section{RECENSIONE:}

\section{Guido PANVINI, Cattolici e violenza politica.} L'altro album di famiglia del terrorismo italiano, Venezia, Marsilio, 2014, 400 pp.

a cura di Jacopo BASSI, Luca BUFARALE *

Cattolici e violenza politica. L'altro album di famiglia del terrorismo italiano si propone di indagare i legami tra il cattolicesimo e la violenza politica. L'autore, Guido Panvini $^{1}$, che ha già affrontato il tema del terrorismo in Italia ${ }^{2}$, lo fa sia esplorando le declinazioni assunte dal pensiero militante cattolico (in grado di spaziare dall'estrema sinistra all'estrema destra), sia indagando - per quanto possibile - l’influenza dei convincimenti religiosi nel determinare le traiettorie biografiche di personalità coinvolte nel terrorismo. Al contesto generale e agli eventi prodottisi in un'era di grandi cambiamenti - in cui, su tutte le vicende, emerge la grande svolta del Concilio Vaticano II - si affiancano le esperienze personali, in grado di gettare una luce sui molti modi di vivere la propria militanza politica e la fede religiosa in quest'epoca.

Panvini apre il suo libro con un prologo che rievoca gli eventi del luglio 1960 a Reggio Emilia: l'autore si sofferma sui portoni chiusi delle chiese - e che non poterono quindi offrire rifugio ai manifestanti comunisti inseguiti dalla polizia - della città emiliana, facendone un simbolo della chiusura di un'epoca - quella del centrismo - e del «[...] passaggio dal pontificato di Pio XII a quello di Giovanni XXIII, nel pieno dei processi di decolonizzazione e con l'incrinatura degli equilibri stabiliti dagli Stati Uniti e dall'Unione Sovietica, nell'imminenza di grandi cambiamenti politici e dello scoppio di un conflitto sociale di vasta portata $[\ldots] \gg 3$.

\footnotetext{
${ }^{1}$ Guido Panvini si è addottorato presso l'Università della Tuscia (dove, attualmente, è docente a contratto) e ha svolto attività di ricerca presso l'Università degli Studi di Macerata e l'Università di Yale.

2 PANVINI, Guido, Ordine nero, guerriglia rossa, Torino, Einaudi, 2009.

3 PANVINI, Guido, Cattolici e violenza politica. L'altro album del terrorismo italiano, Venezia, Marsilio, 2013, p. 23. Il sottotitolo del volume fa riferimento ad un discusso articolo di Rossana
} 
Nel primo capitolo Panvini si sofferma a tratteggiare un quadro d'insieme dei temi dell'antitotalitarismo e dell'anticomunismo in seno al cattolicesimo italiano. Proprio in questo contesto e con l'intento di consolidare un fronte anticomunista nacquero gruppi come Avanguardia Cattolica Italiana - in realtà sorto nel 1919 e attivo fino al 1948 - e si mossero personaggi come Mario Scelba. L'autore sottolinea la necessità di analizzare l'insieme della situazione politica all'inizio degli anni Sessanta per «comprendere il processo di radicalizzazione in seno all'anticomunismo di matrice resistenziale4.

Il secondo capitolo offre un quadro d'insieme del cattolicesimo "di destra" negli anni Sessanta; il focus è sul contesto italiano, ma l'autore ricollega gli eventi al quadro europeo (in particolar modo alla Francia e alla Spagna). Ciò che legava questi gruppi era l'anticomunismo e un'analisi impietosa del liberalismo e, per quel che riguarda l'ambito italiano, delle aperture della Democrazia Cristiana a sinistra. Venivano criticati ferocemente la Democrazia cristiana, partito rappresentativo dei cattolici, e il pontificato di Giovanni XXIII che aveva mancato di condannare in maniera ferma il comunismo e si era mostrato favorevole al processo di decolonizzazione nel Terzo Mondo. Queste posizioni portarono sia alla disobbedienza «legittima» (definita tale perché si riteneva ingiusto l'ordine in materia di fede) di monsignor Lefebvre, sia ad una sorta di psicosi da parte dei cattolici integristi, che si vedevano assediati dal nazionalismo arabo, dall'islam, dai sovietici e, infine, dal laicismo e dalla secolarizzazione che caratterizzavano sempre di più le società occidentali5. All'interno di questo contesto, più voci nel mondo cattolico integrista finirono per legittimare l'uso della violenza come strumento di resistenza nei confronti dei supposti nemici della cristianità.

Nel terzo capitolo l'autore si sofferma sul rapporto tra il mondo cattolico e i fenomeni di decolonizzazione, anche alla luce del Concilio Vaticano II. Quest'ultimo, infatti, rappresentò uno snodo fondamentale nella misura in cui mise in crisi il paradigma che vedeva un'identificazione fra cristianesimo e Occidente, aprì le porte ad un dialogo con il liberalismo e il marxismo e diede nuova spinta alla dottrina sociale della Chiesa e al ruolo attivo dei laici. Sorse così la necessità da un lato di denunciare quello che veniva definito il "neocolonialismo" dei paesi occidentali nei confronti del Terzo Mondo scosso da movimenti di liberazione e, dall'altro, di ripensare il ruolo della stessa fede religiosa, che, nella teologia della liberazione diffusasi specialmente in America Latina tra gli

Rossanda pubblicato sul quotidiano «il Manifesto» il 2 aprile 1978, nel quale si sottolineavano le matrici comuniste del terrorismo di sinistra. Cfr. Ibidem, p. 10.

4 Ibidem, p. 37.

5 Ibidem, p. 115. 
anni Sessanta e Settanta, assunse «una connotazione politica e utopica orientata alla trasformazione dello status quo» 6 :

La riflessione sui movimenti indipendentisti del Terzo Mondo implicò [...] una duplice valutazione. Innanzitutto, la differenza delle condizioni in cui si svolgeva la vita politica in democrazia e nei regimi autoritari comportò l'ammissione della possibile inefficacia, in questi ultimi, dei metodi di lotta non violenti. In secondo luogo, portò a riconoscere nella ribellione armata contro il potere coloniale il medesimo principio morale che aveva legittimato la scelta della Resistenza nei cattolici, in quanto concepita come legittima difesa [...]

Proprio ciò che avveniva in America Latina e in Viet Nam portò allo sviluppo di un dibattito in seno alla Chiesa, preso in esame all'interno del quarto capitolo. La necessità di dare vita a una Chiesa più attenta al dialogo con i poveri (e alle loro esigenze), la consapevolezza che i processi di secolarizzazione e lo sviluppo impetuoso della tecnica stavano sfociando in quello che Del Noce aveva definito un nuovo totalitarismo, volto alla distruzione della religione, le istanze di rinnovamento della società che sarebbero confluite nel Sessantotto, portarono il mondo cattolico ad avviare una riflessione sul tema della violenza (ed in particolare della violenza dei poveri). Panvini rievoca in queste pagine varie figure e diversi movimenti: Don Lorenzo Milani - i cui testi furono spesso fatti propri da una parte della "nuova sinistra" - i "preti operai", l'esperienza dell'abbé Pierre, la rivista «Esprit», gli ambienti della sinistra cattolica della facoltà di sociologia di Trento, sino a Gioventù Studentesca e a Comunione e Liberazione, la cui casa editrice di riferimento, Jaca Book, propose in questi anni una serie di titoli fortemente critici nei confronti del capitalismo, pescando anche nel campo del marxismo eterodosso.

Il capitolo successivo si sofferma sulla visione della violenza politica nel mondo cattolico, vista "da sinistra".

La possibilità per i cristiani di aderire alla rivoluzione rinfocolò il dibattito sulla violenza, spostando l'asse delle discussioni sulle interpretazioni dei passi dell'Antico e del Nuovo Testamento in cui era esplicita l'invocazione al ricorso della forza. Si verificò, inoltre, un significativo slittamento nell'analisi del contesto politico. I dubbi sulle capacità del riformismo di risolvere gli urgenti problemi della società contemporanea si trasformarono, infatti, nella certezza che i progetti

\footnotetext{
${ }^{6}$ Ibidem, p. 195.
}

7 Ibidem, p. 143. 
riformatori avessero ovunque fallito in Occidente. La rivoluzione diveniva, quindi, una possibilità reale, all'interno di un ragionamento in cui le istanze escatologiche si mischiavano con le tensioni politiche ${ }^{8}$.

Panvini approfondisce poi in queste pagine la vicenda politica di Corrado Corghi, segretario della Dc emiliana (partito da cui esce nel 1968) e, per molti versi, paradigmatico del rapporto tra cattolici e violenza politica, in considerazione dei suoi interessi per la guerriglia latinoamericana e dei suoi contatti con alcuni esponenti delle Brigate rosse reggiane.

Il sesto capitolo avvia una riflessione sulla militanza rivoluzionaria e il legame con la fede religiosa. Premessa da parte dell'autore la difficoltà di valutare l'impatto della religione e delle esperienze legate alla fede nel percorso biografico, è da rilevare che:

[...] la fase di transizione apertasi dopo la conclusione del Concilio vaticano II appare uno snodo fondamentale. Sulla scia di questi fermenti nacque una tormentata riflessione sul nesso tra fede, militanza politica e scelta rivoluzionaria. L’inquietudine scaturì dalla constatazione che molte soluzioni al problema della povertà, ipotizzate prima e dopo la protesta studentesca del 1968, erano state inadeguate. Si ritenne, cioè, che la partecipazione dei cristiani alle sofferenze dei poveri non fosse più sufficiente. Era necessario, invece, risolvere tale problema alla radice, non solo partecipando alle lotte dei diseredati, ma ponendosi concretamente l'obiettivo della presa del potere9.

Ed è in queste pagine che Panvini si spinge a tracciare un quadro del mondo cattolico negli anni Settanta e nel 1977 in particolare, prendendo in esame prima le Acli - che, in seguito all'incontro di Vallombrosa del 1970, si avvicinano al Partito socialista - e poi Comunione e Liberazione, in cui l'integrismo in materia religiosa e l'anticomunismo in politica si sposano spesso, in questo periodo, con prese di posizione antiborghesi, antimperialiste e pro-Sessantotto e con un «rapporto ambivalente» nei confronti della violenza $^{10}$, nel tentativo di recuperare terreno tra i giovani colmando il vuoto lasciato dalla crisi delle strutture cattoliche tradizionali e sottraendo consenso alla sinistra extraparlamentare. I paragrafi successivi indagano i percorsi di alcuni affiliati alle Brigate Rosse e ad altri gruppi del terrorismo di sinistra con alle spalle una militanza nelle organizzazioni cattoliche di base, nel volontariato cattolico e, in qualche caso,

\footnotetext{
8 Ibidem, p. 263.

9 Ibidem, pp. 314-315.

10 Ibidem, p. 366.
} 
nella Cisl. L'autore evidenzia come in certe scelte di vita abbia influito l'anelito per un radicale cambiamento nella società, così sentito dai cattolici di sinistra affascinati dalle esperienze di quei sacerdoti dei paesi latinoamericani come Camillo Torres aperti all'opzione della lotta armata contro le dittature.

L'epilogo apre un gioco di sponde con il prologo: per le "porte che si chiudono" richiamate nelle pagine iniziali, l'autore evoca le "porte che si aprono", ovvero la consegna delle armi da parte del gruppo comunista Cocori al segretario del cardinal Martini nel 1984, simbolo del passaggio ad una nuova era.

Corredano il volume un indice delle abbreviazioni e un indice dei nomi, quanto mai opportuno in considerazione della mole di personaggi storici presi in esame.

Il principale merito del libro è di addentrarsi nell'analisi di un filone storiografico in gran parte inesplorato. Finora, infatti, la matrice cattolica di una parte del terrorismo (di sinistra e di destra) è stata poco indagata ${ }^{11}$. Per inquadrare la questione l'autore non manca di fare riferimento ad un'ampia bibliografia che va molto al di là del tema specifico e di prendere in considerazione sia gli studi sul mondo cattolico italiano nelle sue varie declinazioni politiche ${ }^{12}$ sia il dibattito teologico cristiano dagli anni Quaranta in poi, citando autori come Bonhoeffer, Balducci, Del Noce, Quinzio e analizzando varie encicliche papali. Il grande numero di articoli e documenti d'archivio presi in esame costituisce certamente uno dei punti di forza del volume; l'approccio, sempre attento alla spiegazione delle dinamiche generali e del contesto di azione rende la lettura agevole anche per un pubblico di non specialisti. Il libro è quindi un riuscito lavoro di sintesi che ottiene il risultato di offrire un quadro d'insieme di una certa parte del mondo cattolico nelle sue diverse declinazioni politiche e del suo atteggiamento nei confronti della violenza politica durante il secondo dopoguerra.

\footnotetext{
${ }^{11}$ Tra i primi a sostenere, in maniera fortemente polemica, l'importanza della matrice religiosa nel terrorismo di sinistra Panvini cita il giornalista Giorgio Bocca. Cfr. BOCCA, Giorgio, Il terrorismo italiano 1970/1978, Milano, Rizzoli, 1978.

${ }^{12}$ Cfr. ad esempio SARESELLA, Daniela, Cattolici a sinistra. Dal modernismo ai giorni nostri, Roma-Bari, Laterza, 2011; MICCOLI, Giovanni, La Chiesa dell'anticoncilio. I tradizionalisti alla riconquista di Roma, Roma-Bari, Laterza, 2011.
} 


\section{* Gli autori}

Jacopo Bassi è dottorando dell'Università di Genova. Nel corso dei suoi studi si è occupato di storia della Grecia e dell'Albania in età contemporanea e di storia e istituzioni della Chiesa ortodossa. Lavora per le case editrici Il Mulino e Zanichelli.

URL: < http://www.studistorici.com/2009/02/24/jacopo_bassi/ >

Luca Bufarale ha conseguito nel 2008 la Laurea Specialistica in Storia d'Europa (indirizzo contemporaneo) presso l'Università di Bologna e nel 2012 il Dottorato di Ricerca in Scienze storiche presso l'Università di Padova. È autore del volume Riccardo Lombardi. La giovinezza politica 1919-1949 (Roma, Viella, 2014). Attualmente è docente supplente di storia e filosofia nei licei e segue un progetto di ricerca per l'Associazione Labour "Riccardo Lombardi".

URL: < http://www.studistorici.com/2010/12/02/luca-bufarale/ >

\section{Per citare questo articolo:}

BASSI, Jacopo, BUFARALE, Luca, «Recensione: Guido PANVINI, Cattolici e violenza politica. L'altro album di famiglia del terrorismo italiano, Venezia, Marsilio, 2014, 400 pp.», Diacronie. Studi di Storia Contemporanea : La voce del silenzio: intelligence, spionaggio e conflitto nel XX secolo, 29/12/2016,

URL:< http://www.studistorici.com/2016/12/29/bassi-bufarale_numero_28/ >

Diacronie Studi di Storia Contemporanea $\beta$ www.diacronie.it

Risorsa digitale indipendente a carattere storiografico. Uscita trimestrale. redazione.diacronie@hotmail.it

Comitato di redazione: Jacopo Bassi - Luca Bufarale - Antonio César Moreno Cantano - Deborah Paci - Fausto Pietrancosta - Alessandro Salvador - Matteo Tomasoni - Luca Zuccolo

Diritti: gli articoli di Diacronie. Studi di Storia Contemporanea sono pubblicati sotto licenza Creative Commons 3.0. Possono essere riprodotti e modificati a patto di indicare eventuali modifiche dei contenuti, di riconoscere la paternità dell'opera e di condividerla allo stesso modo. La citazione di estratti è comunque sempre autorizzata, nei limiti previsti dalla legge. 